\title{
Erratum: Scattering theory of spin-orbit active adatoms on graphene [Phys. Rev. B 90, 035444 (2014)]
}

\author{
Alexandre Pachoud, Aires Ferreira, B. Özyilmaz, and A. H. Castro Neto \\ (Received 19 June 2015; published 6 July 2015)
}

DOI: 10.1103/PhysRevB.92.039901

PACS number(s): 72.25.-b, 72.80.Vp, 73.20.Hb, 75.30.Hx, 99.10.Cd

Recently, we discovered some mistakes in our article, which have led us to erroneously identify the type of transverse Hall current induced by top-position adatoms on graphene as the "charge Hall effect," instead of a combination of valley Hall effect and spin Hall effect. These errors concern Sec. III C where we investigate the symmetry relations satisfied by coefficients $\mathcal{M}_{s \tau, s \tau}$ and phases $\varphi_{s \tau, s \tau}$ with $s=\uparrow, \downarrow$ and $\tau=K, K^{\prime}$ characterizing the differential cross sections for spin- and valley-preserving scatterings with top-position adatoms. With the notations introduced just above Eqs. (75)-(78) in the original paper, the correct relations are

$$
\begin{gathered}
\mathcal{M}_{2}=\mathcal{M}_{\uparrow K, \uparrow K}^{A}=-\mathcal{M}_{\downarrow K^{\prime}, \downarrow K^{\prime}}^{A}, \\
\mathcal{M}_{0}=\mathcal{M}_{\downarrow K, \downarrow K}^{A}=-\mathcal{M}_{\uparrow K^{\prime}, \uparrow K^{\prime}}^{A}, \\
\varphi_{2}=\varphi_{\uparrow K, \uparrow K}^{A}=\pi-\varphi_{\downarrow K^{\prime}, \downarrow K^{\prime}}^{A}, \\
\varphi_{0}=\varphi_{\downarrow K, \downarrow K}^{A}=\pi-\varphi_{\uparrow K^{\prime}, \uparrow K^{\prime}}^{A} .
\end{gathered}
$$

These relations directly result from Eqs. (25) and (26) of the original paper and are similar to Eqs. (40) and (41) of our paper derived for scattering with hollow-position adatoms in Sec. III B. However, the minus signs and $\pi$ constants were omitted in $\mathrm{Sec}$. III C where the top-position case is addressed. Relations (1)-(4) imply the following symmetries between transverse currents generated by a top-position adatom on sublattice site $\sigma=A(+1), B(-1)$ :

$$
\begin{aligned}
& J_{\uparrow K, \uparrow K}^{\sigma \perp}=-J_{\downarrow K^{\prime}, \downarrow K^{\prime}}^{\sigma \perp}, \\
& J_{\downarrow K, \downarrow K}^{\sigma \perp}=-J_{\uparrow K^{\prime}, \uparrow K^{\prime}}^{\sigma \perp},
\end{aligned}
$$

which in turn imply the existence of charge-neutral transverse valley and spin currents,

$$
\begin{aligned}
& J_{V}^{\sigma \perp}=\sum_{s, \tau} \tau J_{s \tau, s \tau}^{\sigma \perp}=-\sigma \frac{k s_{E} v_{F}}{4}\left(\mathcal{M}_{0} \sin \varphi_{0}+\mathcal{M}_{2} \sin \varphi_{2}\right), \\
& J_{S}^{\sigma \perp}=\sum_{s, \tau} s J_{s \tau, s \tau}^{\sigma \perp}=-\sigma \frac{k s_{E} v_{F}}{4}\left(\mathcal{M}_{2} \sin \varphi_{2}-\mathcal{M}_{0} \sin \varphi_{0}\right) .
\end{aligned}
$$

respectively, and not a transverse charge current as suggested by Eq. (81) of our paper. We note that for the particular case of top-position adatoms without spin-orbit coupling (i.e., $\mathcal{M}_{2}=\mathcal{M}_{0}$ and $\varphi_{2}=\varphi_{0}$ ), the transverse spin current (8) is identically zero, but a nonzero transverse valley current (7) will be induced in general. The remaining equations of Sec. III $\mathrm{C}$ and Fig. 8 together with respective conclusions remain valid upon identification $J_{C}^{\sigma \perp} \rightarrow J_{V}^{\sigma \perp}$.

A.F. acknowledges support from a Royal Society University Research Fellowship. 